\title{
Procuración de tejido corneal y banco de ojos en época de COVID-19 en el estado de San Luis Potosí, México
}

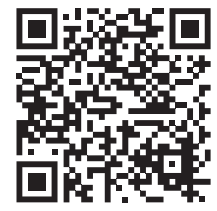

\author{
Procurement of corneal tissue and eye bank in time of \\ COVID-19 in the state of San Luis Potosi, Mexico
}

\author{
Reyna I Tello-Medina, * Thaydeé G Monsiváis-Santoyo, ${ }^{\ddagger}$ Eusermin Gámez-Gómez ${ }^{\ddagger}$ \\ * Cirujana de Trasplante Corneal, responsable del Programa de Trasplante Corneal, Hospital General de Zona 50, IMSS. San Luis Potosí, SLP. \\ ‡ Centro Estatal de Trasplantes. San Luis Potosí, SLP.
}

\section{RESUMEN}

La incidencia de COVID-19 y su mortalidad en el mundo continúa en constante ascenso desafiando a los sistemas de salud, y más aún en el nuestro que se encuentra fragmentado. No existe evidencia de transmisión de ningún coronavirus (SARS-CoV-2, SARS-CoV, MERS) a través de tejido ocular, por lo que las medidas para la transmisión tanto al equipo involucrado en el manejo de la córnea (equipo procurador y personal de banco de ojos) como en el receptor son precautorias, siguiendo lineamientos consensados y cambiantes de índole internacional y nacional. El Centro Nacional de Trasplantes, junto con los integrantes del Subsistema Nacional de Trasplantes y los grupos colegiados de asociaciones científicas, profesionales expertos y organizaciones de la sociedad civil emitieron el Plan Nacional de Reactivación de los programas de Donación y Trasplante, por lo que el actuar ante estas nuevas condiciones de «COVIDANEIDAD» debe mantener la seguridad en todo el personal de salud involucrado en cualquier etapa del proceso.

Palabras clave: COVID-19, coronavirus, SARS-CoV-2, tejido ocular, lineamientos.

\section{INTRODUCCIÓN}

La pandemia causada por el agente viral SARS-CoV-2 ha puesto en evidencia la fragilidad de la salud de la

\section{ABSTRACT}

The incidence of COVID-19 and its mortality worldwide continues to rise, challenging health systems and even more so in ours which is fragmented. There is no evidence of transmission of any coronavirus (SARS-CoV-2, SARS-CoV, MERS) through ocular tissue, so the risk of its transmission to both the team involved in the management of the cornea (team proctor and staff eye bank) and the recipient are precautionary, following agreed and changing international and national guidelines. The National Transplant Center, together with the members of the National Transplant Subsystem and the collegiate groups of scientific associations, professional experts and civil society organizations issued the National Plan for the Reactivation of the Donation and Transplantation programs, so acting before This new "COVIDANEITY» conditions must maintain the safety of all health personnel involved in any stage of the process.

Keywords: COVID-19, coronavirus, SARS-CoV-2, eye tissue, guidelines.

población y lo complicado que puede ser establecer acciones generales efectivas para evitar la propagación y contener la enfermedad. La incidencia de COVID-19 y su mortalidad en todo el mundo continúan

Citar como: Tello-Medina RI, Monsiváis-Santoyo TG, Gámez-Gómez E. Procuración de tejido corneal y banco de ojos en época de COVID-19 en el estado de San Luis Potosí, México. Rev Mex Traspl. 2021; 10 (1): 29-33. https://dx.doi.org/10.35366/99849 
en constante ascenso. Los desafíos que enfrentan los sistemas de salud son múltiples, particularmente en nuestro país con un sistema de salud fragmentado.

En la actualidad, no existe evidencia de la transmisión del SARS-CoV-2, MERS o cualquier otro coronavirus a través del tejido ocular, ${ }^{1-3}$ al contrario, existe evidencia que demuestra ausencia del SARS-CoV-2 en la córnea, ${ }^{4}$ por lo que el riesgo en relación al equipo involucrado en el manejo del tejido ocular se considera bajo y las medidas son precautorias; sin embargo, se han publicado estudios que demuestran presencia de receptores de la enzima convertidora de angiotensina 2 (ECA2) en órganos humanos, incluyendo la superficie de la córnea y la conjuntiva, ${ }^{5}$ actuando como punto de entrada al organismo, por lo que garantizar la protección del equipo procurador del tejido corneal, así como del personal involucrado en el manejo de estos tejidos dentro del Banco de Ojos deberá realizarse atendiendo medidas de protección estandarizadas y consensadas en orden mundial, apegado a las recomendaciones de asociaciones internacionales, así como de los lineamientos nacionales.

El Centro Nacional de Trasplantes en coordinación con los integrantes del Subsistema Nacional de Trasplantes, representados por las instituciones de salud, gobiernos de las entidades federativas a través de los centros y consejos estatales de trasplantes, grupos colegiados de asociaciones científicas, profesionales expertos y organizaciones de la sociedad civil, han emitido el Plan Nacional para la Reactivación de los programas de Donación y Trasplantes, basado en semaforización de las condiciones epidemiológicas locales y de cada establecimiento, mismas que se analizan y se actualizan regularmente dependiendo del comportamiento de la pandemia. ${ }^{6,7}$

El presente documento tiene como objetivo proporcionar una guía al personal de salud involucrado en el trasplante de córnea para realizar los procedimientos de forma segura, minimizando riesgos tanto para los receptores del trasplante, como para el equipo de trasplantes y personal de salud involucrado.

El estado de San Luis Potosí cuenta con 11 programas activos de trasplantes de córnea distribuidos en instituciones públicas y privadas, con un promedio de 147.8 queratoplastias anuales; durante los últimos cinco años se ha posicionado como el segundo tipo de trasplante más frecuente en la entidad.

Se encuentran registrados para trasplante de córnea 5,657 en todo el país, en el estado de SLP son 86 pacientes inscritos (37 mujeres y 49 hombres) a la espera del reinicio de los programas de trasplante corneal.
El origen del tejido corneal para trasplante proviene de donante fallecido, lo que reitera la necesidad de reactivar los programas de donación cadavérica, para lo cual se deben considerar los siguientes contextos:

Semaforización de riesgo epidemiológico de la entidad federativa: emitida por la Secretaría de Salud, basada en los indicadores de proporción (a la ocupación hospitalaria y a la positividad del virus) y a los indicadores de tendencia (en casos hospitalizados y del síndrome COVID-19). ${ }^{6,7}$

Semaforización de riesgo epidemiológico de los establecimientos: su generación y actualización es responsabilidad del comité interno de trasplantes de cada establecimiento, el cual identifica el riesgo epidemiológico de la entidad federativa y la situación particular en cada hospital, institución y/o establecimiento de salud, así como la capacidad para el funcionamiento de los programas de donación y trasplantes ${ }^{4,8}$ basándose en las siguientes variables:

1. Situación epidemiológica de circulación del virus SARS-CoV-2 (COVID-19).

2. Circuitos libres de COVID-19.

3. Disponibilidad de camas de hospitalización y en unidades de cuidados intensivos (UCI).

4. Estudios de laboratorio y gabinete: pruebas PCR, tomografía axial computarizada (TAC).

5. Personal de salud: disponibilidad de equipo de protección personal (EPP), personal sin rotación por áreas COVID-19, pruebas PCR.

6. Participación de comités hospitalarios de bioética y unidades de vigilancia epidemiológica.

7. Consentimiento informado para COVID-19.

\section{Selección de donante de tejido corneal:3,6,7,9-11}

1. Se deberá excluir donantes con PCR-TR (transcriptasa reversa-reacción en cadena de la polimerasa) positivo a SARS-CoV-2 o con diagnóstico clínico de COVID-19.

2. Se deberá excluir donantes con enfermedad respiratoria aguda o fiebre o con al menos un síntoma severo o común asociado a COVID-19 (Tabla 1).

3. Se excluye el caso si no se dispone de prueba confirmatoria por PCR.

4. Se excluyen casos con sospecha clínica de COVID-19 aunque exista prueba PCR negativa.

5. Se excluyen donantes que hayan estado expuestos a un caso confirmado o sospechoso de COVID-19 en los últimos 14 días. 
6. Se excluyen los casos con causa de muerte indeterminada.

7. Se excluirán potenciales donantes con riesgo epidemiológico (exposición a un caso confirmado de COVID-19 o viaje/residencia en áreas afectadas en los 21 días previos).

8. Se excluirán potenciales donantes con evidencia de conjuntivitis.

9. Se excluirán pacientes con síndrome de distrés respiratorio agudo (SDRA), neumonía evidenciada por tomografía computada que muestre la típica imagen de «opacidades en vidrio esmerilado».

\section{Precauciones para el equipo procurador de cor- neas: $6,9,10$}

1. Documentar el riesgo del potencial donante mediante interrogatorio intencionado y una historia clínica relevante a algún miembro de la familia, asegurando que todo el personal del hospital generador y del banco estén informados del caso.

2. Es obligatorio procurar el tejido corneal con la precaución de que se haya expuesto el tejido ocular previamente a la doble exposición de yodopovidona $10 \%$ (por al menos 6 minutos) antes de su procuración por el efecto viricida que se ha demostrado in vitro, lo anterior forma parte del procedimiento habitual de desinfección o inactivación microbiana que está validado para virus envueltos. Sin embargo, se desconoce si las partículas de virus infecciosos están presentes dentro de las células de la superficie ocular o dentro de las capas más profundas del tejido ocular que pueden o no ser eliminadas por las preparaciones de povidona yodada. ${ }^{11}$

3. Usar equipo de protección personal (EPP) consistente en mascarilla N95, gorro quirúrgico, protector

Tabla 1: Síntomas de COVID-19.11

\begin{tabular}{lll}
\hline Severos & Comunes & Menos comunes \\
\hline Disnea & Fiebre & Mialgias \\
Dolor torácico & Tos seca & Fatiga \\
Confusión & Disnea leve & Cefalea \\
Labios y cara azulada & & Hemoptisis \\
Cianosis & Diarrea \\
& Odinofagia \\
& Rinorrea \\
& Tos húmeda \\
& Pérdida de gusto y olfato \\
\hline
\end{tabular}

facial/visor, guantes y bata quirúrgica que deberán ser usados mientras se procura el tejido ocular.

EI EPP se debe remover después de la procuración y empacarse apropiadamente para evitar infección cruzada, y se desecha en las bolsas correspondientes de los residuos peligrosos biológicos infecciosos. Es necesario que las partes no desechables del EPP, como las gafas protectoras o visor, se limpien con hipoclorito de sodio al $1 \%$ al retirarse el equipo. Es recomendable contar con capacitación en el entrenamiento del uso del EPP.

4. Utilización de bolsas dobles para las muestras de sangre y del frasco de conservación de la córnea, asegurando resistencia adecuada y evitar fugas.

5. El personal del equipo procurador que extrae los tejidos y maneja los materiales e instrumentales debe lavar sus manos con agua y jabón por arriba del codo durante aproximadamente 30 segundos antes de cualquier otro procedimiento. ${ }^{9}$

\section{Recomendaciones para los bancos de ojos: $:^{6,9,12}$}

1. Vigilar que la calidad de los tejidos cumpla con los estándares internacionales establecidos para garantizar las condiciones óptimas del trasplante; así como verificar que los equipos procuradores hayan ejecutado las acciones necesarias para la calidad del tejido, teniendo en consideración la situación hospitalaria del hospital generador.

2. El tejido corneal que entra al banco de ojos debe provenir de un hospital libre de COVID-19, o de un hospital híbrido donde no se compartió áreas con pacientes COVID-19 (área libre de COVID-19) en ningún momento durante la hospitalización del donante.

3. Todo tejido ingresado al banco de ojos requiere estudio de microbiología (cultivo) con la finalidad de minimizar el riesgo de patógenos bacterianos y mantener en "cuarentena" hasta contar con el resultado de la prueba de PCR negativa a SARSCoV-2 antes de ser liberado para su uso terapéutico, y en caso de ser positiva se desecha el tejido bajo el procedimiento establecido para tal fin.

4. Al recibir los tejidos remitidos para su resguardo temporal de los establecimientos de salud autorizados, el banco de ojos deberá limitar el riesgo de contaminación mediante las siguientes medidas:

a. Limpiar exhaustivamente todas las superficies externas y el equipo de embalaje, bote del medio de preservación, paquetes de hielo, instrumental quirúrgico y caja contenedora del instru- 
mental con alcohol al $70 \%$ o preparado fresco de hipoclorito de sodio $1 \%$. $^{8,13}$

b. El piso deberá limpiarse con hipoclorito de sodio $1 \%$ cada dos horas.

c. Si hay contaminación del área deberá realizarse una limpieza profunda.

d. Las manijas, puertas, barandales y superficie de alto contacto como escritorio, sillón, sala, deberá limpiarse con hipoclorito de sodio al 1\% cuatro veces al día.

e. Las sillas en la sala de espera, equipo electrónico, monitor, tablero, mouse y demás periféricos expuestos deberán ser higienizados con toallas en alcohol cada dos horas.

f. El equipo de protección del personal en el banco de ojos deberá incluir mascarilla N95 u otra mascarilla aprobada como son las mascarillas tricapa quirúrgicas, guantes, protección facial y bata regular. Los bancos de ojos deberán asegurarse de contar con una reserva suficiente de estos equipos de protección y material sanitizante.

g. Realizar el lavado de manos de manera frecuente y usar sanitizantes de manos usualmente durante la jornada laboral.

h. La mascarilla es obligatoria en presencia de otras personas.

i. Mantener la separación en las estaciones de trabajo, distancia física mínima de $1.5 \mathrm{~m}$ entre el personal.

j. Deberá realizarse tamizaje de riesgo y síntomas al personal para el ingreso a las instalaciones. En caso de síntomas sugestivos de COVID-19 o exposición de riesgo se canalizará al servicio médico para valoración y diagnóstico. Durante el periodo de sospecha deberá evitar el ingreso al banco de ojos. Para los casos COVID-19 confirmados se requerirá mantener aislamiento, el reingreso a las instalaciones será después de 14 días, al contar con prueba PCR negativa a SARS-CoV-2.

5. Todo el expediente del donante y documentos serán expuestos a luz ultravioleta en la campana de flujo laminar por 30 minutos inmediatamente después de que el equipo procurador llega al banco de ojos (30 minutos de cada lado de las hojas). ${ }^{9}$

6. La limpieza del microscopio especular deberá ser exhaustiva posterior a cada proceso de valoración.

7. Los bancos de córneas únicamente podrán distribuir los tejidos a los establecimientos de salud autorizados para trasplante corneal con los que se tengan celebrados convenios para tal efecto y que cuenten con las condiciones necesarias para realizar el trasplante, contemplados en el Plan Nacional de Reactivación con conocimiento de las autoridades nacionales y estatales en la materia.,12

8. Mantener en óptimas condiciones el tejido en espacios seguros libres de COVID-19 y otros posibles agentes contaminantes, al término de la caducidad del medio de preservación intermedio es necesario cambiar el tejido a glicerina con la finalidad de mantener por más tiempo el tejido y que se use para fines de asignación prioritaria (la glicerina cuenta con propiedades inhibitorias de crecimiento bacteriano y actividad antiproteasas, además de fungir como agente deshidratante manteniendo la estructura corneal). Por último, lo que se pretende con esto es tener técnicas de conservación alternativas para mejorar la resiliencia frente a las inevitables amenazas infecciosas futuras. ${ }^{14,15}$

9. Mantenerse actualizados de acuerdo con la normativa mexicana, los manuales internos de procedimientos, incluyendo las estrategias que se deben ejecutar disminuyendo riesgos inherentes por el COVID-19. ${ }^{12}$

\section{CONCLUSIONES}

La córnea es el tejido con mayor demanda a nivel nacional y estatal para su uso terapéutico, por lo que es indispensable continuar con los programas de donación y trasplante de córnea, así como con el entrenamiento del personal de salud involucrado, desde el uso del equipo de protección personal hasta la obtención del tejido corneal con técnicas depuradas y la capacitación en el manejo del tejido en el Banco de Ojos con todas las medidas sanitarias que se requieren de acuerdo con las recomendaciones y protocolos emitidos por las organizaciones nacionales e internacionales de trasplantes, con la finalidad de disminuir el contagio y la transmisión de la enfermedad de COVID-19, garantizando la calidad y la seguridad en el tejido liberado para el trasplante.

La pandemia por SARS-CoV-2 afectó de manera significativa los programas de donación y trasplantes de órganos y tejidos en todo el mundo, particularmente en México, y sus efectos pueden ser duraderos ya que los hospitales públicos y privados reconvertidos para la atención de pacientes COVID-19 representan un alto riesgo de infección para la actividad de donación y trasplantes, y los casos que requieren un órgano o tejido se enfrentarán a una larga espera por la escasez 
de donantes aunado a la situación cambiante, que nos hará consensar frecuentemente las recomendaciones para implementar estrategias que permitan limitar el riesgo y dar respuesta a la necesidad apremiante de órganos y tejidos, de allí la importancia de restablecer esta actividad para atender la demanda.

La procuración de tejido corneal tiene un grado de complejidad importante y más en estos tiempos, donde se requiere disponibilidad de recursos humanos, materiales y financieros. El reto que tenemos es continuar promoviendo la cultura de la donación para incrementar y mantener la aceptación a la donación, optimizar tiempos, pruebas de laboratorio (PCR) y pronta entrega de resultados, todo esto con la finalidad de no prolongar los tiempos en la procuración y en la atención a deudos.

En el estado de San Luis Potosí se gestiona la reactivación de los programas de trasplante de tejido corneal basándonos en un manual interno de procedimiento con lineamientos técnicos actualizados en relación a la pandemia de COVID-19, con el análisis correspondiente a la conservación de los tejidos cuya finalidad es garantizar la calidad de éstos, cumpliendo con los requisitos de infraestructura, equipamiento y control sanitario para continuar con la actividad del Plan Nacional de Reactivación, , $, 7,12$ beneficiando así a los casos registrados en espera de trasplante, minimizando todo tipo de riesgo durante esta pandemia debida al SARS-CoV-2.

\section{REFERENCIAS}

1. Centros para el Control y la Prevención de Enfermedades (EE.UU). Nuevo coronavirus 2019. Disponible en: https:// www.cdc.gov/coronavirus/COVID-2019/hcp/clinical-criteria. html?CDC_AA_ref Val=https $\% 3 \mathrm{~A} \% 2 \mathrm{~F} \% 2 \mathrm{Fwww} . \mathrm{cdc}$. gov\%2Fcoronavirus\%2FCOVID-2019\%2Fclinical-criteria.html

2. Bayyoud T, Iftner A, Iftner T, Bartz-Schmidt KU, Rohrbach JM, Ueffing $\mathrm{M}$ et al. Absence of severe acute respiratory syndromecoronavirus-2 rna in human corneal tissues. Cornea. 2021; 40 (3): 342-347. doi: 10.1097/ICO.0000000000002479.

3. Tello-Medina RI, Verdiguel-Sotelo K. Consideraciones para la donación y trasplante de tejido ocular en México durante la pandemia de COVID-19. Rev Mex Traspl. 2020; 9 (Suppl: 2): 216-221. doi:10.35366/94511.

4. US Food \& Drug Administration, US Department of Health \& Human Services. Updated information for human cell, tissue, or cellular or tissue-based product (HCT/P) establishments regarding the COVID-19 pandemic. 2 July 2020. Available in: https://www.fda.gov/vaccines-blood-biologics/safety-availabilitybiologics/updated-information-human-cell-tissue-or-cellular-ortissue-based-product-hctp-establishments.

5. Gupta PC, Kumar MP, Ram J. COVID-19 pandemic from an ophthalmology point of view. Indian J Med Res. 2020; 151 (5): 411-418.

6. Secretaría de Salud/Centro Nacional de Trasplantes. Plan de reactivación de los programas de donación y trasplantes [actualización 11 de agosto de 2020].

7. Aburto-Morales JS, Romero-Méndez J, Lucio-García CA, Madrigal-Bustamante JA. México ante la epidemia de COVID-19 (SARS-CoV-2) y las recomendaciones al Subsistema Nacional de Donación y Trasplante. Rev Mex Traspl. 2020; 9 (1): 6-14.

8. WHO. Infection prevention and control during health care when novel coronavirus (nCoV) infection is suspected. Geneva: WHO; 2020. Interim guidance. 25 January 2020. Available in: https:// covid-19.conacyt.mx/jspui/bitstream/1000/5134/1/1108313.pdf

9. Eye Bank Association of America. Information about COVID-19 (coronavirus) is being released rapidly. we are posting updates here as we get them. These recommendations have been updated as of July 31, 2020. [Consulta 16 de agosto de 2020] Available in: https://restoresight.org/?s=coronavirus

10. Sharma N, D'Souza S, Nathawat R, Sinha R, Gokhale NS, Fogla R et al. All India Ophthalmological Society - Eye Bank Association of India consensus statement on guidelines for cornea and eyebanking during COVID-19 era. Indian J Ophthalmol. 2020; 68 (7): 1258-1262.

11. COVID-19 Update Guidance and screening Recommendations by Eye Bank Association of America date 14 Abril 2020. [Acceso 16 de agosto de 2020] Available in: http.//restoresight.org/covid19-updates/.

12. Reglamento de la Ley General de Salud en Materia de Trasplantes. Nuevo Reglamento publicado en el Diario Oficial de la Federación el 26 de marzo de 2014. Disponible en: http:// www.diputados.gob.mx/LeyesBiblio/regley/Reg_LGS_MT.pdf

13. Kampf G, Todt D, Pfaender S, Steinmann E. Persistence of coronaviruses on inanimate surfaces and their inactivation with biocidal agents. J Hosp Infect. 2020; 104 (3): 246-251.

14. Busin M, Yu AC, Ponzin D. Coping with COVID-19: an italian perspective on corneal surgery and eye banking in the time of a pandemic and beyond. Ophthalmology. 2020; 127 (9): e68-e69.

15. Gupta N, Dhasmana R, Maitreya A, Badahur H. Glycerolpreserved corneal tissue in emergency corneal transplantation: An alternative for fresh corneal tissue in COVID-19 crisis. Indian J Ophthalmol. 2020; 68 (7): 1412-1416.

\section{Correspondencia:}

Dra. Reyna I Tello-Medina

Programa de Trasplante de Córnea, Hospital General de Zona 50,

IMSS, San Luis Potosí.

E-mail: dratellocornea@yahoo.com 